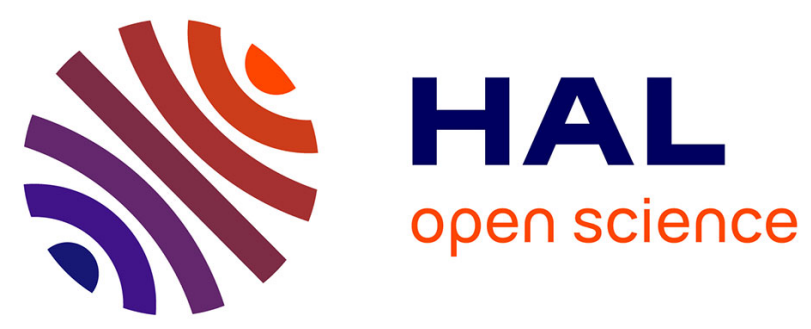

\title{
Improvement of the Digital Image Correlation close to the borders of an object
}

Maxime Baconnais, Julien Réthoré, Marc François

\section{To cite this version:}

Maxime Baconnais, Julien Réthoré, Marc François. Improvement of the Digital Image Correlation close to the borders of an object. Strain, 2020, 56 (3), 10.1111/str.12340 . hal-02920373

\section{HAL Id: hal-02920373 \\ https://hal.science/hal-02920373}

Submitted on 24 Aug 2020

HAL is a multi-disciplinary open access archive for the deposit and dissemination of scientific research documents, whether they are published or not. The documents may come from teaching and research institutions in France or abroad, or from public or private research centers.
L'archive ouverte pluridisciplinaire HAL, est destinée au dépôt et à la diffusion de documents scientifiques de niveau recherche, publiés ou non, émanant des établissements d'enseignement et de recherche français ou étrangers, des laboratoires publics ou privés. 


\title{
Improvement of the Digital Image Correlation close to the borders of an object
}

\author{
Maxime Baconnais, Julien Réthoré, Marc François \\ Université de Nantes, Centrale Nantes, CNRS, GeM, UMR 6183, F-44000 Nantes, France
}

13 February 2020

\begin{abstract}
The measurement error of the finite-element based Digital Image Correlation (DIC) is reduced along the borders of an object by using the precise measurement, obtained by Virtual Image Correlation (VIC). The proposed method is called Virtual and Digital Image Correlation (VDIC). The boundary, identified by the VIC with a sub-pixel precision, is used firstly to create an adapted mesh and secondly to create a pixel mask. Futhermore, the VDIC also uses the VIC measurement of the boundary in the deformed state as a constraint on the radial displacement field along the border. The optimal values of the parameters of the VDIC are discussed throughout a sensitivity analysis. The compared performances of the constrained and unconstrained VDIC are obtained thanks to a synthetic test of a plate with a hole in tension. Finally, the method is checked on a sample geometry which includes holes and a U-shaped notch.
\end{abstract}

keywords: digital image correlation, virtual image correlation, boundary displacement, pixel mask, adapted mesh

\section{Notations}

- $F$ reference state image

- $G$ deformed state image

- $F^{*}$ virtual image related to $F$

- $f_{o}$ and $f_{b}$ respectively foreground (object) and background gray levels of the virtual image

- $\delta F$ boundary of the object in $F$, as detected by VIC

- $\delta G$ boundary of the object in $G$, as detected by VIC

- $\boldsymbol{n}$ local normal to the boundary

- $\boldsymbol{X}$ position vector in the frame of the reference image

- $\boldsymbol{X}_{\delta F}$ position vector of a point of the boundary $\delta F$

- $\boldsymbol{X}_{\delta G}$ position vector of a point of the boundary $\delta G$

- $\boldsymbol{U}$ motion vector from reference to deformed image

- $\boldsymbol{U}^{\text {ref }}$ exact motion vector from reference to deformed image

- $\lambda_{k}$ DIC set of motion parameters

- $\boldsymbol{N}_{k}$ DIC finite element shape functions 
- $\Psi$ DIC cost function

- $\mu_{k}$ VIC set of shape parameters

- $\Phi$ VIC cost function

- $c_{b}$ pixel exclusion criterion

- $\alpha$ constraint level

- $\Psi_{G}$ mean distance from the deformed border $\delta F$ to $\delta G$

\section{Introduction}

Nowadays, Digital Image Correlation (DIC) is the most used full-field measurement technique in research and industrial contexts, due to its capacity to measure displacements with good accuracy and resolution. Furthermore, this approach needs a minimal experimental setup: a camera, paint, and lighting. DIC is used for a large range of applications: high-resolution measurement [2, cracks detection [15], in extended dimension for out-of-plane displacements measurement with stereo-correlation [9, 13] or for 3D measurement with tomography images [15. Since the early 80s, many improvements have been developed to increase accuracy and resolution. However, in particular zones, this method cannot accurately measure the displacement and strain fields [18. Oscillations appear on the boundary, which consists of the initial borders of the sample but also of new borders generated by possible cracks. Whereas, in mechanics, free boundaries hold essential information. This systematic error is due to discontinuities in the displacement field, between the sample and the background or between two separate parts of the same sample [19, 3. The classical technique to avoid this problem is to limit the region of interest inside the sample surface and to exclude its boundary from the analysis. However, measuring the displacement as close as possible to the boundary is important, and new approaches have been developed by using an adapted mesh and by removing from the computation, using a mask, the pixels located in the background [19, 4].

The purpose of this communication is to use an accurate boundary measurement to constrain the solution of the DIC, within an existing DIC code [16, in order to obtain the same precision close to the boundary and far from it. The method for detecting the position of the boundary is the Virtual Image Correlation (VIC) [14, 8, 6, 7, which is able to detect the position of a boundary in a digital image with better accuracy than computer graphics issued methods [5, 17]. The principle of the VIC is to find the best correlation between a virtual image and the contour. The virtual image consists in a white to black progression in the vicinity of a parametric curve which is, in this article, a generic B-Spline.

This article is separated into four parts. In Section 2, Digital Image Correlation and Virtual Image Correlation are summarized. In Section 3, the proposed Virtual and Digital Image Correlation (VDIC) is introduced. In Section 4 the influence of the parameters of the method, constraint level and pixel mask criterion, are evaluated. Also, a study of an infinite plate in tension with synthetic images is performed to compare the new method to existing ones. Then, in Section 5, an experimental test is used to compare the different correlation methods on real cases, whose results are finally discussed in Section 6 .

\section{Summary of the DIC and VIC methods}

\subsection{Measurement of the displacement field with DIC}

The DIC method requires two images: the "reference" one F and the "deformed" one G, of the same speckle applied on the surface of the deformable object. The gray levels are represented respectively by $F(\boldsymbol{X})$ and $G(\boldsymbol{X})$ where $\boldsymbol{X} \in \mathbb{N}^{2}$ are the pixels coordinates. The displacement field $\boldsymbol{U}(\boldsymbol{X})$, which is searched for the best correlation between $\mathrm{F}$ and $\mathrm{G}$.

Following the optical flow equation, the reference and deformed images are related through

$$
F(\boldsymbol{X})=G(\boldsymbol{X}+\boldsymbol{U}(\boldsymbol{X}))
$$


In this article, a global DIC method [3] is used, i.e. the full-field is evaluated at each coordinate through the minimization of a unique cost function $\Psi(\boldsymbol{U})$ which consists in a sum of squared differences:

$$
\Psi(\boldsymbol{U})=\int_{\Omega}[G(\boldsymbol{X}+\boldsymbol{U}(\boldsymbol{X}))-F(\boldsymbol{X})]^{2} \mathrm{~d} \boldsymbol{X},
$$

where $\Omega$ is the region of interest defined in the reference image.

Mathematically, $\boldsymbol{U}(\boldsymbol{X})$ cannot be estimated for each pixel coordinate $\boldsymbol{X}$ because it represents two unknowns, $u_{X_{1}}$ and $u_{X_{2}}$, for only one gray level data: $G(\boldsymbol{X}+\boldsymbol{U})-F(\boldsymbol{X})$. Therefore the displacement field is defined from a parameterized kinematic model $\boldsymbol{U}\left(\boldsymbol{X}, \lambda_{k}\right)$, where $\lambda=\left\{\lambda_{1}, \ldots, \lambda_{m}\right\}$ are the unknown kinematic parameters. In this article, the simple finite-element basis which allows to study a lot of mechanical problems is used [1]:

$$
\boldsymbol{U}(\boldsymbol{X}, \lambda)=\sum_{k=1}^{m} \lambda_{k} \boldsymbol{N}_{k}(\boldsymbol{X})
$$

where $\lambda_{k}$ are the nodal displacements along the two directions $X_{1}$ and $X_{2}$, and where $\boldsymbol{N}_{k}(\boldsymbol{X})$ are the interpolation functions.

To solve the DIC problem, the deformed image is assumed to be differentiable. Thus, the updated deformed image, due to an incremental displacement field $\Delta \boldsymbol{U}(\boldsymbol{X})$, is

$$
G(\boldsymbol{X}+\boldsymbol{U}+\Delta \boldsymbol{U})=G(\boldsymbol{X}+\boldsymbol{U})+\Delta \boldsymbol{U} \cdot \boldsymbol{\nabla} G(\boldsymbol{X}+\boldsymbol{U}) .
$$

Hence, the minimization condition $\partial \Psi(\boldsymbol{U}+\Delta \boldsymbol{U}) / \partial \Delta \lambda_{l}=0$ leads to the linear system

$$
\int_{\Omega}[F(\boldsymbol{X})-G(\boldsymbol{X}+\boldsymbol{U})]\left[\boldsymbol{N}_{l} \cdot \nabla G\right] \mathrm{d} \boldsymbol{X}=\sum_{k=1}^{m} \Delta \lambda_{k} \int_{\Omega}\left[\boldsymbol{N}_{k} \cdot \nabla G\right]\left[\boldsymbol{N}_{l} \cdot \nabla G\right] \mathrm{d} \boldsymbol{X}
$$

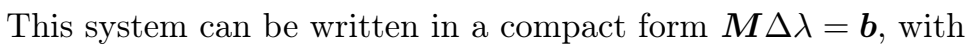

$$
\begin{aligned}
& M_{l k}=\int_{\Omega}\left[\boldsymbol{N}_{k} \cdot \nabla F\right]\left[\boldsymbol{N}_{l} \cdot \nabla F\right] \mathrm{d} \boldsymbol{X}, \\
& b_{l}=\int_{\Omega}[F(\boldsymbol{X})-G(\boldsymbol{X}+\boldsymbol{U})]\left[\boldsymbol{N}_{l} \cdot \boldsymbol{\nabla} F\right] \mathrm{d} \boldsymbol{X} .
\end{aligned}
$$

The gradient $\boldsymbol{\nabla} G(\boldsymbol{X}+\boldsymbol{U}(\boldsymbol{X}))$ is replaced by $\boldsymbol{\nabla} F(\boldsymbol{X})$ because this approximation can be made in most case [11] and $\boldsymbol{\nabla} F(\boldsymbol{X})$ can be calculated once. Thus, with an initial guess $\boldsymbol{U}^{0}$, the linear system enables the iterative computation of the displacement field $\boldsymbol{U}^{i+1}=\boldsymbol{U}^{i}+\Delta \boldsymbol{U}^{i}$ until convergence.

In this system, the gradient $\nabla F$ is one of the most important terms. Hence, the method needs a gray level texture, called speckle, in the images. Moreover, to avoid local minima issues, this speckle has to be random.

\subsection{Measurement of the boundary position with VIC}

Virtual Image Correlation (VIC) [17] [14] is also an image correlation method, like the DIC. However, it is not aimed at measuring the displacement field between two digital images, but at detecting the position of the boundary of an object in a unique image $F$. For this purpose, a virtual image $F^{*}$ is created, quite similar to the boundary to be detected. An algorithm close to the one used for DIC is adopted to determine the position and the shape of this created image that best matches the reference image.

The VIC determines an analytical description of the boundary geometry in the digital image. To define the shape of the virtual image - in the coordinate system $\boldsymbol{X}$ of the digital image - a parametric curve model $\delta F$ is chosen. It depends on a curvilinear coordinate $x_{1} \in[0,1]$ and some shape parameters $\mu=\left\{\mu_{1}, \ldots, \mu_{m}\right\}$ whose final values represent the VIC measurement. This curve may correspond to many descriptors like polygon, circle, ellipse, B-Spline, NURBS... 
In a digital image, a boundary corresponds to a continuous variation of the gray level within a narrow pixel band. Indeed, for the pixel in which passes the boundary, the gray level depends on the fraction of the photons which comes from the sample surface and from the background. Therefore, a thickness $2 R$ is defined to extend the curve $\delta F$ to a $2 \mathrm{D}$ object. The virtual image is then represented by several points of definition:

$$
\boldsymbol{X}\left(x_{1}, x_{2}, \mu_{k}\right)=\boldsymbol{X}_{\delta F}\left(x_{1}, \mu_{k}\right)+x_{2} R \boldsymbol{n}\left(x_{1}, \mu_{k}\right),
$$

where $\boldsymbol{X}_{\delta F}\left(x_{1}, \mu_{k}\right)$ denotes the current points along $\delta F, x_{2} \in[-1,1]$ the second local coordinate of the virtual image and $\boldsymbol{n}$ the local normal to $\delta F$. For a sharp image, the optimal thickness is $R=2$ pixels [5].

Finally, the gray level of the virtual image is created. Its distribution is defined as a function of the signed distance $x_{2}$ between the pixel center and the curve in the local system $\left(x_{1}, x_{2}\right)$ :

$$
F^{*}\left(x_{1}, x_{2}, \mu_{k}\right)=F^{*}\left(x_{2}\right)=\frac{\left(f_{b}-f_{o}\right) x_{2}+f_{b}+f_{o}}{2} .
$$

This distribution can be adapted to the object and background average gray levels, called respectively $f_{o}$ and $f_{b}$.

As for DIC, the parameters $\mu_{k}$ are evaluated through the minimization of a cost function defined by a least-squares residual. This function $\Phi(\mu)$ writes

$$
\Phi(\mu)=\frac{1}{2} \int_{0}^{1} \int_{-1}^{1}\left[F^{*}\left(x_{2}\right)-F\left(\boldsymbol{X}\left(x_{1}, x_{2}, \mu_{k}\right)\right)\right]^{2} \mathrm{~d} x_{2} \mathrm{~d} x_{1} .
$$

This quadratic function can be minimized with a Newton-Raphson algorithm [17] where, at each iteration, the increment of the parameters $\Delta \mu$ is calculated by

$$
\frac{\partial \Phi^{2}}{\partial \mu_{l} \partial \mu_{k}} \Delta \mu_{k}=-\frac{\partial \Phi}{\partial \mu_{l}}
$$

This system can be rewritten in a compact and simplified form $\boldsymbol{M \Delta} \mu=\boldsymbol{b}$, with

$$
\begin{aligned}
& M_{l k}=\int_{\Omega^{*}}\left(\frac{\partial \boldsymbol{X}}{\partial \mu_{k}} \cdot \nabla F\right)\left(\frac{\partial \boldsymbol{X}}{\partial \mu_{l}} \cdot \nabla F\right) \mathrm{d} \boldsymbol{X}, \\
& b_{l}=-\int_{\Omega^{*}}\left[F^{*}-F\right]\left(\frac{\partial \boldsymbol{X}}{\partial \mu_{l}} \cdot \nabla F\right) \mathrm{d} \boldsymbol{X},
\end{aligned}
$$

with here $F=F\left(\boldsymbol{X}\left(x_{1}, x_{2}, \mu_{k}\right)\right)$ the digital image interpolated on the computation points of the virtual one. The numerical discretization of $\left(x_{1}, x_{2}\right)$ is chosen such as the distance between two computational points in the pixel frame is less than 1 pixel. From an initial set $\mu_{k}^{0}$, the parameters are iteratively evaluated by

$\mu_{k}^{l+1}=\mu_{k}^{l}+\Delta \mu_{k}^{l}$ until convergence. Because of the regularity of the parametric curve model, the accuracy can be close to $1 / 1000$ pixel [17, 5].

\section{$3 \quad$ VIC to help DIC}

The strategy adopted for the Virtual and Digital Image Correlation (VDIC) relies on four steps:

1. VIC measurement of the boundaries $\delta F$ in $\mathrm{F}$ and $\delta G$ in $\mathrm{G}$,

2. creation of an adapted mesh, whose boundary nodes are on $\delta F$,

3. generation of a pixel mask, eliminating pixels closer than $c_{b}$ from $\delta F$,

4. resolution of the DIC problem, constrained by the final location of boundary nodes which must belong to $\delta G$, with constraint level $\alpha$. 


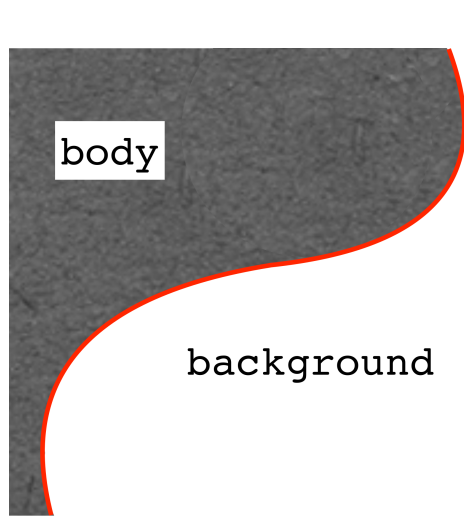

(a)

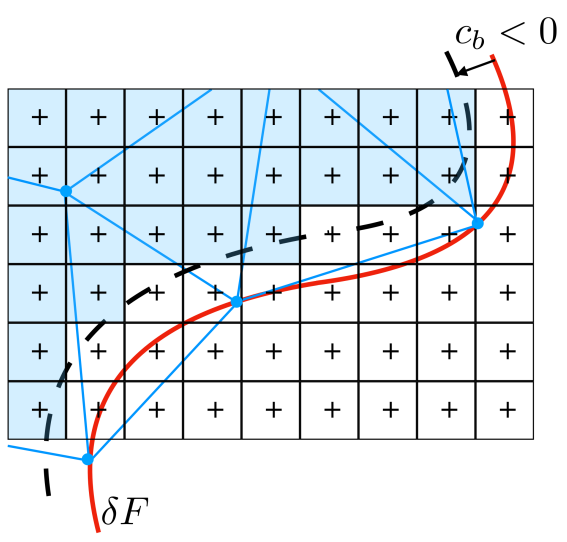

(b)

Figure 1: Sketch of the method: measured position of the boundary of the object (a), adapted mesh and pixel mask illustration (b).

Figure 1 shows a boundary $\delta F$ identified by VIC and the finite element nodes which are precisely located on $\delta F$. The mask is defined as follows: the pixels (in white) whose center is outside a parallel curve distant of $c_{b}$ from $\delta F$ are rejected. In the constrained VDIC, the finite element nodes are, similarly, forced to belong to the curve $\delta G$.

Step 1 is clear from Section 2.2. Step 2 is easy when using relevant constrained meshing methods such as 12. Step 3 requires the computation of the signed distance from the current point $\boldsymbol{X}$ to the boundary $\delta F$ :

$$
\left(\boldsymbol{X}-\boldsymbol{X}_{\delta F}\right) \cdot \boldsymbol{n}<c_{b},
$$

where $\boldsymbol{X}_{\delta F}$ is the projection of $\boldsymbol{X}$ on $\delta F$, along the outward pointing normal $\boldsymbol{n}$. If $c_{b}>0$, only the pixels whose center is located outside of $\delta F$ might removed. On the contrary, setting a negative value defines an exclusion band along the border. Especially, setting $-\sqrt{2} / 2 \leq c_{b} \leq \sqrt{2} / 2$ excludes any pixel intersecting $\delta F$. The influence of $c_{b}$ on the precision will be discussed in Section 4.2 . Step 4 requires to define a distance from the border points in the deformed configuration, $\boldsymbol{X}+\boldsymbol{U}$, to the border $\delta G$ identified in G:

$$
\Psi_{G}=\int_{\delta G}\left(\boldsymbol{X}+\boldsymbol{U}-\boldsymbol{X}_{\delta G}\right)^{2} d \boldsymbol{X}_{\delta F} .
$$

In this expression $\boldsymbol{X}_{\delta G}$ is the projection of $\boldsymbol{X}+\boldsymbol{U}$ on $\delta G$. In a numerical point of view, this projection is defined by the point of $\delta G$ which minimizes its distance from $\boldsymbol{X}+\boldsymbol{U}$. However, the (subpixel) discretization of $\delta F$ and $\delta G$ induces a slight tangential component in the vector $\boldsymbol{X}+\boldsymbol{U}-\boldsymbol{X}_{\delta G}$. Thus, from a practical point of view, the theoretically equivalent definition is retained:

$$
\Psi_{G}=\int_{\delta G}\left[\left(\boldsymbol{X}+\boldsymbol{U}-\boldsymbol{X}_{\delta G}\right) \cdot \boldsymbol{n}\right]^{2} d \boldsymbol{X}_{\delta F} .
$$

When $\Psi_{G}=0$, the nodes which belong to the boundary $\delta F$ at the initial state also belong to the boundary $\delta G$ in the deformed state. This constraint defines their radial motion $U_{r}$ but lets their tangential motion $U_{t}$ free and only defined by the DIC. The proposed method consists in defining a global cost function which is a weighted balance between the classical DIC function $\Psi$ and the above function $\Psi_{G}$ :

$$
\Pi=\Psi+A \Psi_{G},
$$

where $A$ is a parameter of the method. Setting $A=0$ consists in using the DIC with an adapted mesh (Steps 1 and 2) and a pixel mask of width $c_{b}$ (step 3). In this case, there is no need to identify $\delta G$ with the VIC, only the identification of $\delta F$ is necessary. Setting a higher value for $A$ corresponds to impose the nodes of the boundary to belong precisely to the boundary $\delta G$. The stationarity condition of $\Pi$ is:

$$
\frac{\partial \Pi(\boldsymbol{U}+\Delta \boldsymbol{U})}{\partial \Delta \lambda_{l}}=\frac{\partial \Psi(\boldsymbol{U}+\Delta \boldsymbol{U})}{\partial \Delta \lambda_{l}}+A \frac{\partial \Psi_{G}(\boldsymbol{U}+\Delta \boldsymbol{U})}{\partial \Delta \lambda_{l}}=0,
$$


with the derivative of $\Psi_{G}$ :

$$
\left.\frac{\partial \Psi_{G}}{\partial \Delta \lambda_{l}}=2 \int_{\partial \delta F}\left[\left(\boldsymbol{X}+\boldsymbol{U}+\sum_{k=1}^{m} \Delta \lambda_{k} \boldsymbol{N}_{k}(\boldsymbol{X})-\boldsymbol{X}_{\delta G}\right)\right) \cdot \boldsymbol{n}\right]\left[\boldsymbol{N}_{l}(\boldsymbol{X}) \cdot \boldsymbol{n}\right] \mathrm{d} \boldsymbol{X}_{\delta F}
$$

Equation (17) leads, like Equation (4) for the DIC problem, to find iteratively $\Delta \lambda$ from the resolution of the linear system

$$
(M+A C) \Delta \lambda=b+A d,
$$

where

$$
\begin{aligned}
& C_{l k}=\int_{\delta G}\left[\boldsymbol{N}_{l} \cdot \boldsymbol{n}\right]\left[\boldsymbol{N}_{k} \cdot \boldsymbol{n}\right] \mathrm{d} \boldsymbol{X}_{\delta F}, \\
& d_{l}=\int_{\delta G}\left[\left(\boldsymbol{X}+\boldsymbol{U}-\boldsymbol{X}_{\delta G}\right) \cdot \boldsymbol{n}\right]\left[\boldsymbol{N}_{l} \cdot \boldsymbol{n}\right] \mathrm{d} \boldsymbol{X}_{\delta F} .
\end{aligned}
$$

However, $\Psi$ and $\Psi_{G}$ are not comparable because $\Psi$ is a quadratic mean of gray levels and $\Psi_{G}$ depends upon a distance in pixels. Thus, $A$ has to be defined independently for each case. For this reason, the VDIC problem is normalized with respect to the operators $M$ and $C$ as follows:

$$
\left(\frac{M}{\|M\|}+\alpha \frac{C}{\|C\|}\right) \Delta \lambda=\left(\frac{b}{\|M\|}+\alpha \frac{d}{\|C\|}\right),
$$

where $\alpha$ replaces $A$ as the second parameter of the method but is intrinsic and whose value will be discussed in the next section.

As recalled previously, the DIC method requires a speckle on the correlation domain. However, the presented VDIC method has to distinguish, for the VIC (step 1), the background from the speckle. For this reason, the dynamic range of the speckle has been reduced in the following simulations and tests. For example, in the synthetic sketch image 1 (see Figure 2a), the dynamic contrast of the speckle is [0 - 128] and the background is at level 255. This reduction of the dynamic range is a cost to pay for using VDIC. The VIC is then used with adapted background and foreground virtual images gray levels $f_{b}$ and $f_{o}$.

\section{Parameter sensitivity analysis}

The VDIC method presented above uses two parameters: $\alpha$, for the relative weight of DIC and VIC, which influences the radial position of the nodes of the boundary, and $c_{b}$, which defines the width of the pixel exclusion band around the boundary. For this study, two set of $256 \times 256$ pixels synthetic images $\mathrm{F}$ and $\mathrm{G}$ have been created. Test 1 consists in an object with a circular hole, deformed by a rigid body motion: $U_{x}^{\text {ref }}=0.5$ pixel, $U_{y}^{\text {ref }}=0.5$ pixel. Test 2 consists in an object with a hole whose shape is a B-Spline of degree 2, deformed by a linear displacement field: $U_{x}^{\text {ref }}=x / 256$ pixel, $U_{y}^{\text {ref }}=y / 512$ pixel. The retained linear FE basis is obviously theoretically able to fit the prescribed linear displacement field. The VDIC method is used for both tests by using 16 pixel triangular linear finite elements for the DIC part and the optimal curves (circle and B-Spline) with a final width $R=1.5$ pixel for the VIC part.

The synthetic images are synthesized as follows:

- A hundred times refined binary image is created, which contains randomly distributed black spots over a white background.

- A boundary is created from an analytical curve (straight line, B-Spline, circle...). The domain outside of this boundary is replaced by a uniform white backgroun.

- The synthetic image at the final resolution is computed from the density of subpixel binary image, mimicking an ideal pixel whose response is proportional to the quantity of light it receives.

- Finally the dynamic range of the object is reduced to [0 128].

This procedure leads to realistic blurred edges (see Figure $2 \mathrm{a}$ for example), of width $\simeq 1$ pixel, around spots and around the borders $\delta F$. Image $G$ is created in a very similar way, the location of subpixels being moved according to some known (reference) field denoted as $\boldsymbol{U}^{\text {ref }}$. The precision of the measurement is obtained from the comparison between the measured, by DIC or VDIC methods, displacement field $\boldsymbol{U}$ and $\boldsymbol{U}^{\text {ref }}$, in the narrow band where the VDIC can give different results from the DIC. 


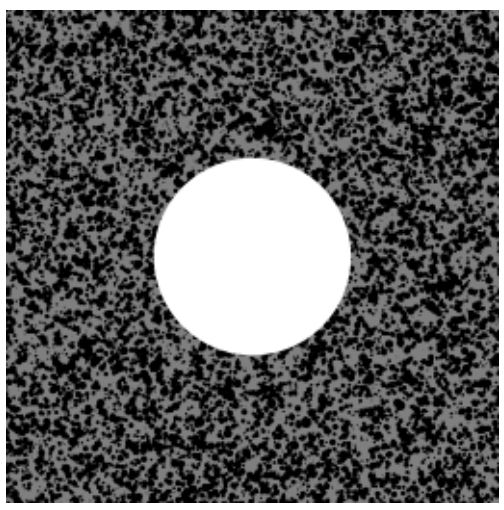

(a)

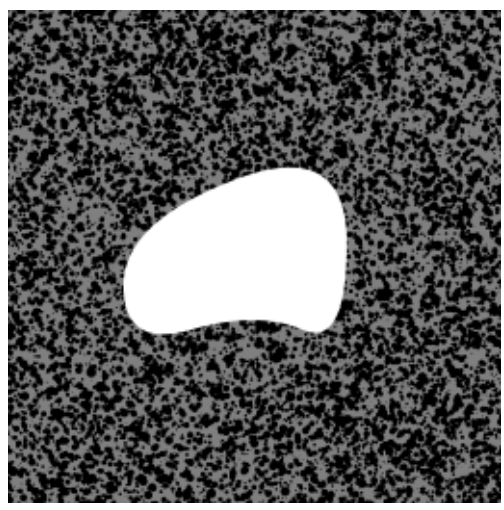

(b)

Figure 2: Reference images $\mathrm{F}$ for the test 1 (a) and the test 2 (b).

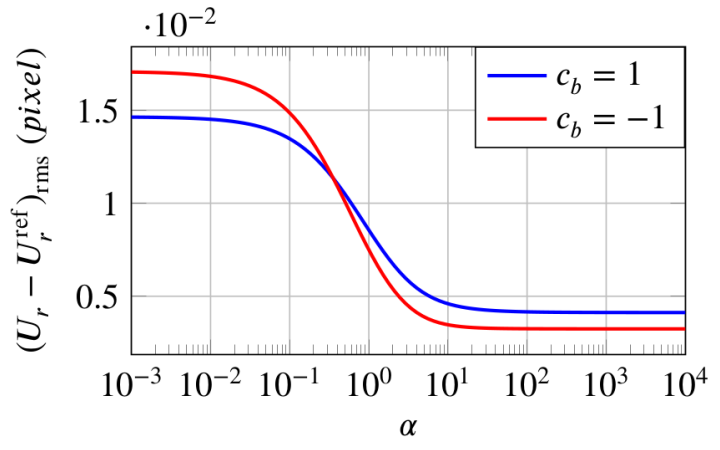

(a)

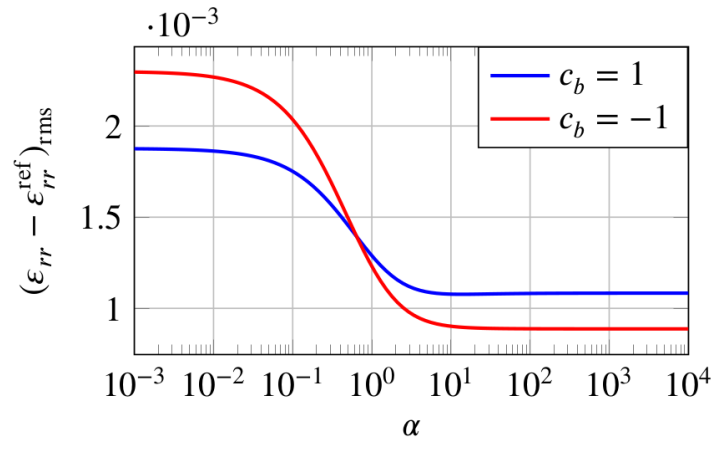

(b)

Figure 3: RMS value, for the test 1, of the radial displacement (a) and strain (b) error as a function of $\alpha$, computed within a band of 8 pixel.

\subsection{Influence of the parameter $\alpha$}

The remaining parameter $c_{b}$ is fixed at two key values, 1 and -1 , which correspond respectively to a strict inclusion and a strict exclusion of any pixel which contains $\delta F$. The parameter $\alpha$ influences the way the nodes are constrained to belong to the contour $\delta G$ at the deformed state. Figure 3 respectively Figure 4. shows the RMS error for test 1 , respectively test 2 , of the radial displacement $\left(U_{r}-U_{r}^{\text {ref }}\right)_{\text {rms }}$ and the radial strain $\left(\varepsilon_{e r}-\varepsilon_{r r}^{\text {ref }}\right)_{\text {rms }}$ as a function of $\alpha$. The error is computed in a pixel band of 8 pixels, half the size of the elements size. In both cases, Figure 3 and Figure 4 show that a value of $\alpha=50$ is enough to get the best precision on the radial displacement and strain on the boundary, and that the VDIC is close to twice more precise than the DIC for this criterion. The saturation observed for large $\alpha$ corresponds to the cases when the node belongs precisely to the contour $\delta G$. Comparable results have been obtained on various test cases, with other sizes of images and meshes. This light overshoot, visible in Figure 4 for test 2, has no generality. Very high values of $\alpha$ should be avoided because, firstly, they do not provide better precision and, secondly, they lead the system, presented in Equation 22, to be ill-conditioned. The advantage of the VDIC is to set more or less precisely (depending upon $\alpha$ ) the radial position of the finite element nodes.

\subsection{Influence of the parameter $c_{b}$}

The parameter $c_{b}$ defines the pixel mask, more precisely the width of the band around $\delta F$ in which pixels are not taken into account. Negative values of $c_{b}$ induce a loss of information, reducing the number of significant pixels in the finite elements close to the boundary, but are often required in practical cases (as seen in the next sections) when the boundary includes physical or optical defects. The tangential displacements $U_{t}$ are 


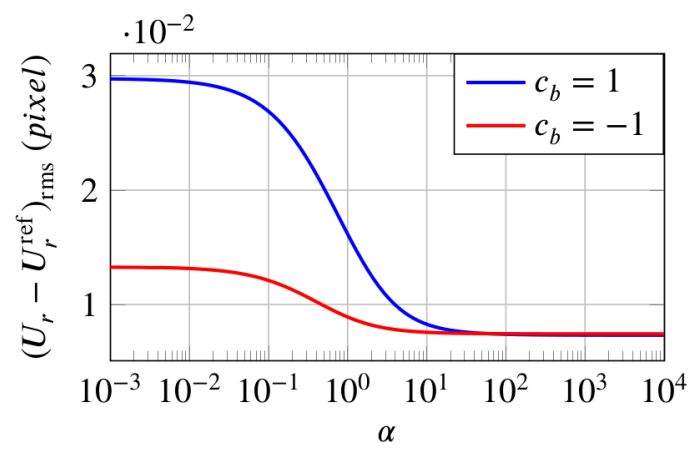

(a)

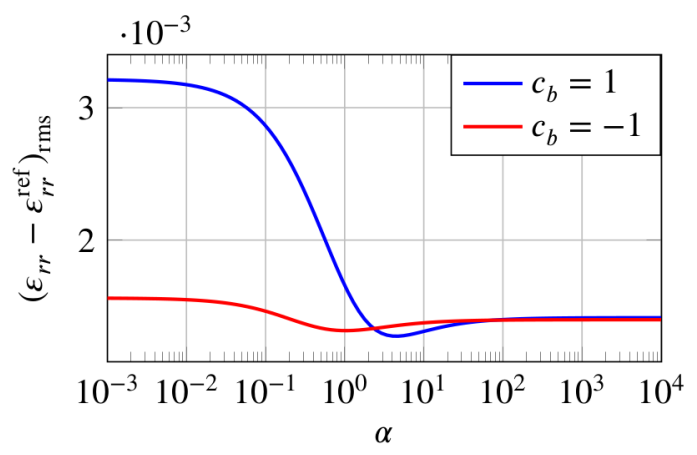

(b)

Figure 4: RMS value, for the test 2, of the radial displacement (a) and strain (b) error as a function of $\alpha$, computed within a band of 8 pixel.

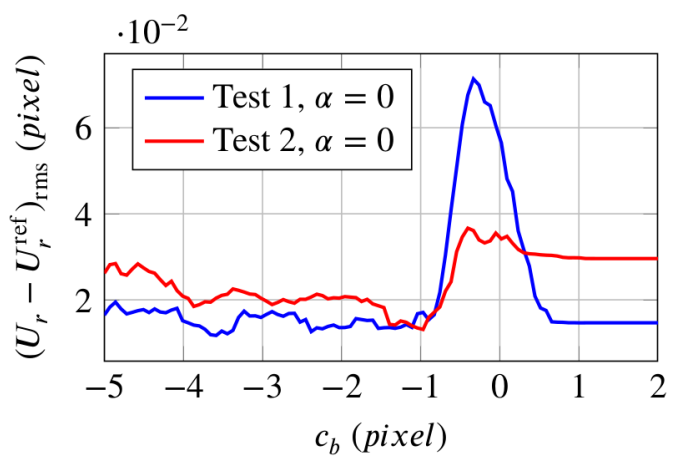

(a)

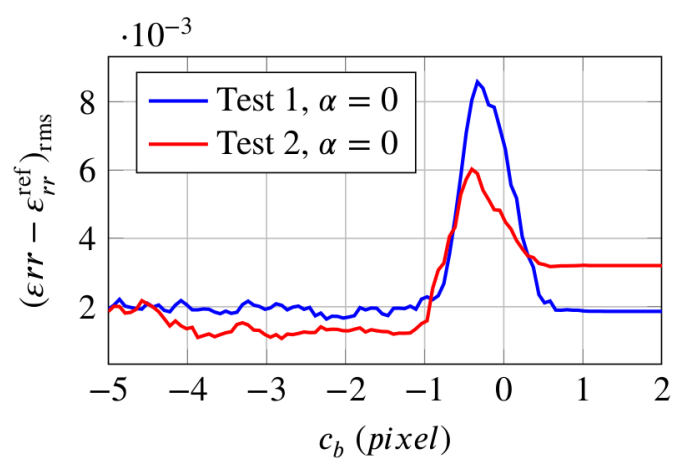

(b)

Figure 5: RMS value of the radial displacement (a) and strain (b) error as a function of $c_{b}$, computed on a band of width 8 pixels.

not constrained and are unaffected by $c_{b}$, as long as the loss of information is not too high, i.e. $-c_{b}<<L_{e}$, where $L_{e}$ is the finite elements average size. In the case of constrained VDIC, the radial displacements $U_{r}$ are defined by the boundary position $\delta G$ and are also unaffected by $c_{b}$. This was confirmed by many tests. However, in the case of unconstrained VDIC $(\alpha=0)$, Figure 5 shows that the radial displacements $U_{r}$ and strain $\varepsilon_{r r}$ depend upon $c_{b}$ for both test cases.

Positive values of $c_{b}$ lead to take into account the gray level of the background. Thus, in these cases of ideal images with a uniformly white background with a null gradient, this justifies the plateau for $c_{b}>1$. For negative values of $c_{b}$, Figure 5 shows a slight increase of the error induced by the growing number of suppressed significant pixels. Figure 5 also shows that $c_{b}=0$ is generally not a good choice. Furthermore, it shows that a strict exclusion $\left(c_{b}=-1\right)$ leads to equal or better results than the strict inclusion $\left(c_{b}=1\right)$ (in this case of perfect background). In practical cases where the border and background are not perfect, one should set $c_{b} \leq-1$, the final choice depending upon the distance from the border that can be affected by burrs, paint drop, diffraction, etc.

\subsection{Performance test}

The performance of the VDIC is evaluated hereafter on the example of an infinite plate in tension (along the $x$ axis), with a circular hole. Such type of problems will doubtless constitute one of the major applications of the VDIC in the field of mechanical testing. The stress and the displacement fields are given by [10]: 


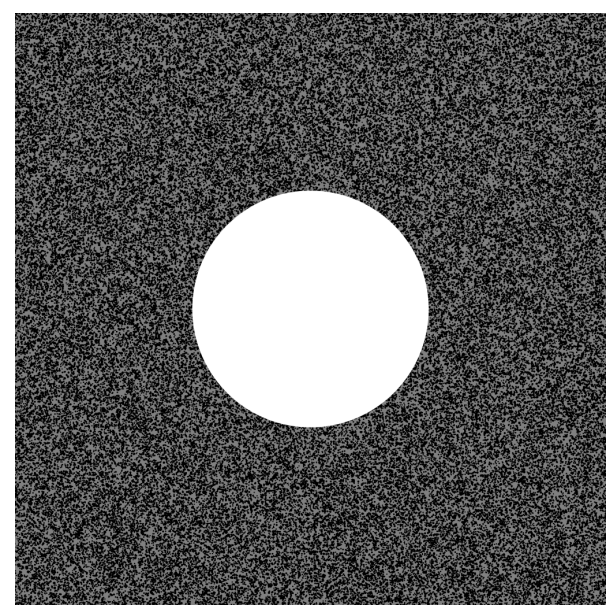

Figure 6: Plate in tension along the $x$ axis with a hole: reference image $F$.

$$
\begin{gathered}
\sigma_{r r}=\frac{\sigma^{\infty}}{2}\left(1-\frac{a^{2}}{r^{2}}\right)+\frac{\sigma^{\infty}}{2}\left(1+\frac{3 a^{4}}{r^{4}}-\frac{4 a^{2}}{r^{2}}\right) \cos 2 \theta, \\
\sigma_{\theta \theta}=\frac{\sigma^{\infty}}{2}\left(1+\frac{a^{2}}{r^{2}}\right)-\frac{\sigma^{\infty}}{2}\left(1+\frac{3 a^{4}}{r^{4}}\right) \cos 2 \theta, \\
\sigma_{r \theta}=-\frac{\sigma^{\infty}}{2}\left(1-\frac{3 a^{4}}{r^{4}}+\frac{2 a^{2}}{r^{2}}\right) \sin 2 \theta, \\
u_{r}=\frac{\sigma^{\infty}}{2 E}\left[(1+\nu)\left(\frac{a^{2}}{r}+\left(r-\frac{a^{4}}{r^{3}}\right) \cos 2 \theta\right)+(1-\nu) r+\frac{4 a^{2}}{r} \cos 2 \theta\right], \\
u_{\theta}=-\frac{\sigma^{\infty}}{2 E}\left[(1+\nu)\left(1+\frac{a^{4}}{r^{4}}\right) r+(1-\nu) \frac{2 a^{2}}{r}\right] \sin 2 \theta,
\end{gathered}
$$

where $a$ is the hole radius, $E$ the Young modulus, $\nu$ Poisson's ratio, $(r, \theta)$ the polar coordinates and $\sigma^{\infty}$ the stress $\sigma_{x x}$ at infinity. The magnitude of the displacements is $\sigma^{\infty} / E=0.0039$. The $1024 \times 1024$ pixels images are synthesized for both initial $F$ (see Figure 6) and final $G$ states.

The measurement is realized with the VDIC in three ways:

- Unconstrained and unmasked VDIC with $\alpha=0$ and $c_{b}=1$.

- Unconstrained and masked VDIC with $\alpha=0$ and $c_{b}=-1$.

- Constrained and masked VDIC with $\alpha=50$ and $c_{b}=-1$.

In all cases, the (initially) circular hole border is measured by VIC using a B-Spline curve of order 3 and 14 control points. This choice of curve has been validated by a simulation using the exact analytical initial and final shapes of the hole, which proved to give very close results. For the DIC part, the triangular and linear finite elements have $L_{e}=40$ pixels far from the hole, and $L_{e}=20$ pixels in the vicinity of the hole. The uncertainty on the identification is revealed by the map of the norm of the displacement error $\left\|\boldsymbol{U}-\boldsymbol{U}^{\text {ref }}\right\|$ shown in Figure 7.

A careful look at the error along the hole border shows that the constrained and masked identification gives better results along the boundary, as expected. Furthermore, a difference of these maps shows that the measure is identical for a distance greater than $2 L_{e}$ from the boundary. The unconstrained and masked VDIC - not presented in Figure 7 - gives similar results. Table 1 and 2 show the bias and uncertainty (RMS) for both radial and tangential displacements, calculated in the band of width $2 L_{e}$ around the hole. Table 3 shows the bias and uncertainty for radial strain.

These results show that the masked and moreover the constrained VDIC increases the precision of the 


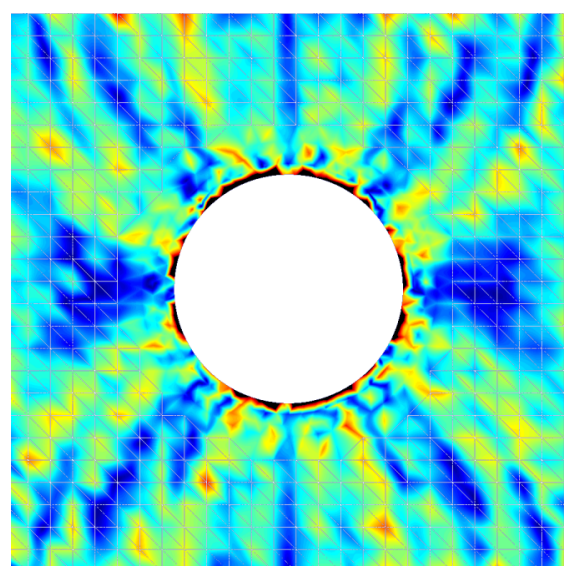

(a) $\left(\alpha=0, c_{b}=1\right)$

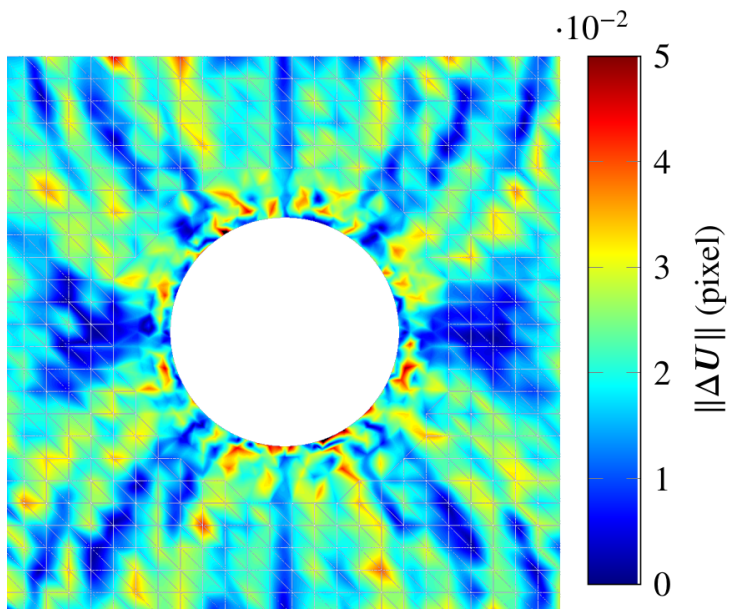

(b) $\alpha=50, c_{b}=-1$ )

Figure 7: Map of the error in displacement for the tensile simulation on a circular hole (Figure 6). Unconstrained and unmasked VDIC $\alpha=0, c_{b}=1$ (a), and constrained and masked VIDC $\alpha=50, c_{b}=-1$ (b).

\begin{tabular}{l|c|c}
$\Delta U_{r}$ (pixel) & Bias & Uncertainty \\
\hline Unconstrained and unmasked, $\alpha=0$ and $c_{b}=1$ & 0.0132 & 0.0251 \\
Unconstrained and masked, $\alpha=0$ and $c_{b}=-1$ & 0.0073 & 0.0165 \\
Constrained and masked, $\alpha=50$ and $c_{b}=-1$ & 0.0066 & 0.0157
\end{tabular}

Table 1: Bias and uncertainty values of $\Delta U_{r}$ on the boundary.

\begin{tabular}{l|c|c}
$\Delta U_{t}$ (pixel) & Bias & Uncertainty \\
\hline Unconstrained and unmasked, $\alpha=0$ and $c_{b}=1$ & 0.0006 & 0.0167 \\
Unconstrained and masked, $\alpha=0$ and $c_{b}=-1$ & 0.0005 & 0.0169 \\
Constrained and masked, $\alpha=50$ and $c_{b}=-1$ & 0.0005 & 0.0169
\end{tabular}

Table 2: Bias and uncertainty values of $\Delta U_{t}$ on the boundary.

\begin{tabular}{l|c|c}
$\Delta \varepsilon_{r r}$ & Bias & Uncertainty \\
\hline Unconstrained and unmasked, $\alpha=0$ and $c_{b}=1$ & 0.0023 & 0.0040 \\
Unconstrained and masked, $\alpha=0$ and $c_{b}=-1$ & 0.0009 & 0.0018 \\
Constrained and masked, $\alpha=50$ and $c_{b}=-1$ & 0.0007 & 0.0016
\end{tabular}

Table 3: Bias and uncertainty values of $\Delta \varepsilon_{r r}$ on the boundary. 


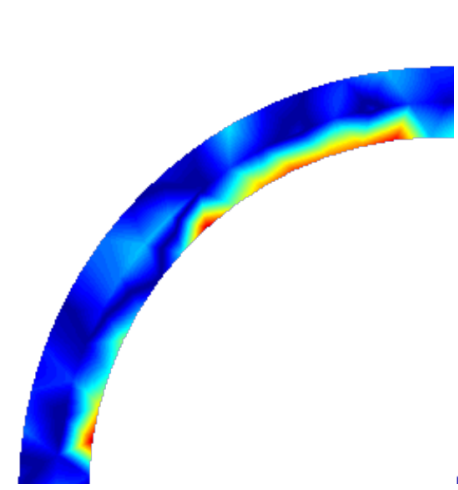

(a) $\left(\alpha=0, c_{b}=1\right)$

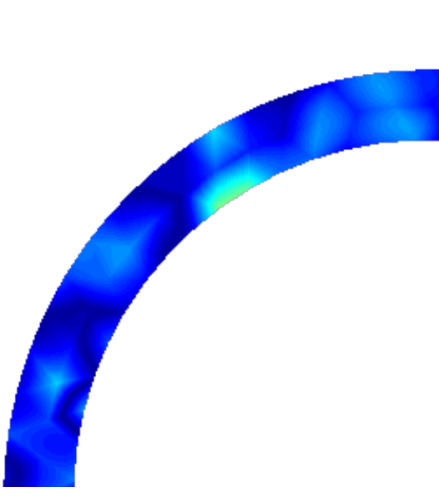

(b) $\left(\alpha=0, c_{b}=-1\right)$

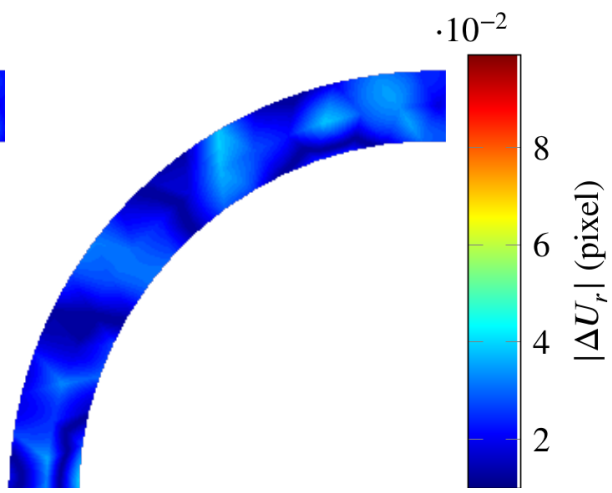

(c) $\left(\alpha=50, c_{b}=-1\right)$

Figure 8: Error in radial displacement $\left|\Delta U_{r}\right|$ for the plate in tension with a hole, in the band of width $2 L_{e}$.

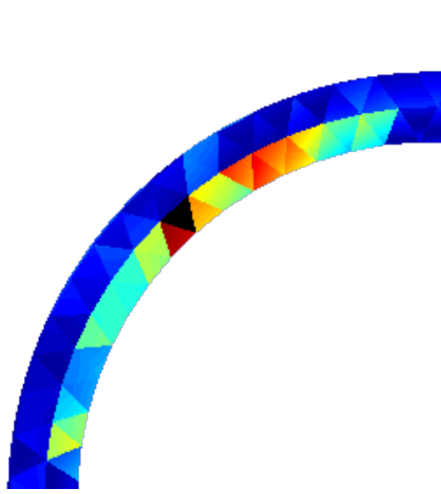

(a) $\left(\alpha=0, c_{b}=1\right)$

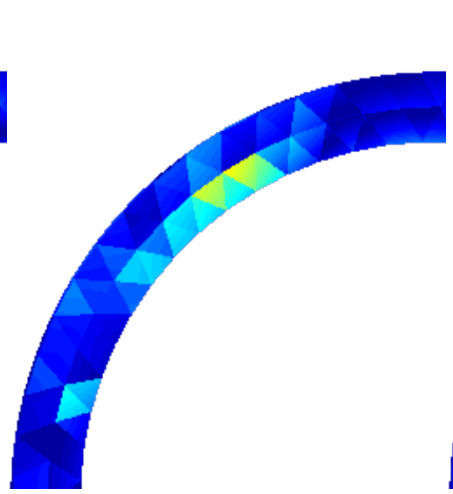

(b) $\left(\alpha=0, c_{b}=-1\right)$

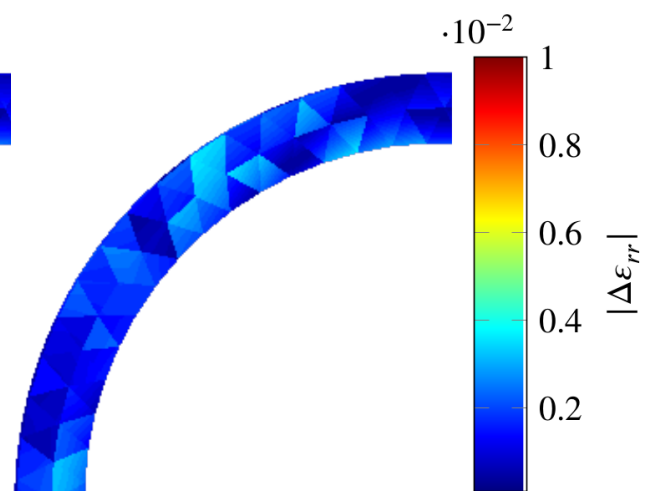

(c) $\left(\alpha=50, c_{b}=-1\right)$

Figure 9: Error in radial strain $\left|\Delta \varepsilon_{r r}\right|$ for the plate in tension with a hole, in the band of width $2 L_{e}$.

measurement of the radial displacement and strain. On the contrary, the tangential displacement is, logically, not improved but also quite not depreciated. Figures 8 and 9 present the radial displacement and strain, around the hole for the three VDIC methods. For clarity, only the data in the band of $2 L_{e}$, and for the top left quarter of the plate, are represented. These results show that the gain in precision of the masked and constrained identification is concentrated close to the border, this being the first objective of the method. Furthermore, the constrained VDIC reduces the local measurement error remaining with the masked and unconstrained VDIC.

\section{Application}

This test is relative to an experiment similar to the Sandia fracture challenge which consists in a tearing test with opposed motions of both side of the upper part of the plate, as shown in Figure 10a

The image is in 8 bits, black and white, and of size $6576 \times 4384$ pixels.

The two images have been taken at the very beginning of the test, thus, the displacement field is supposed to be a rigid body motion due to the play in the testing device. A careful view of Figure 10b shows that, certainly due to diffraction or diffusion effects, the speckle has a lighter color close to the boundaries. Furthermore, many paint drops are visible on this test which was not prepared specifically for VDIC but for classical DIC measurement.

The retained parameters were:

- DIC settings: element size $L_{e}=50$ pixels. 


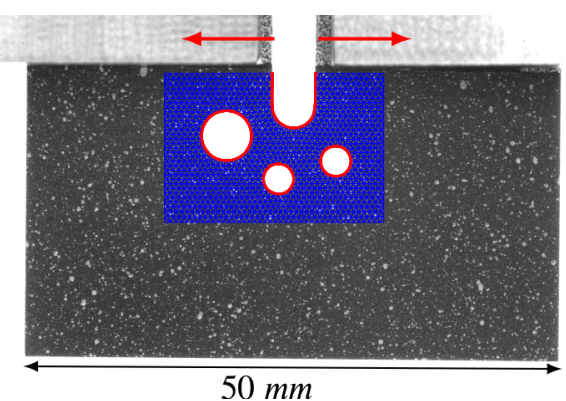

(a)

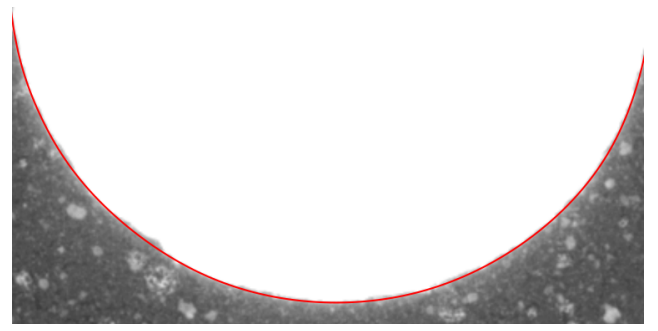

(b)

Figure 10: Reference image of the tearing test with adapted mesh and initial boundary position $\delta F$ (a). Zoom on the U shaped notch and VIC measurement (b).

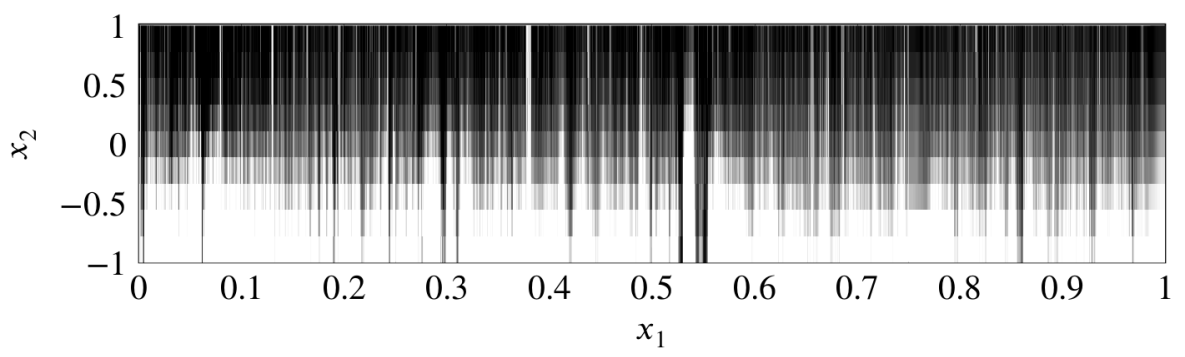

(a)

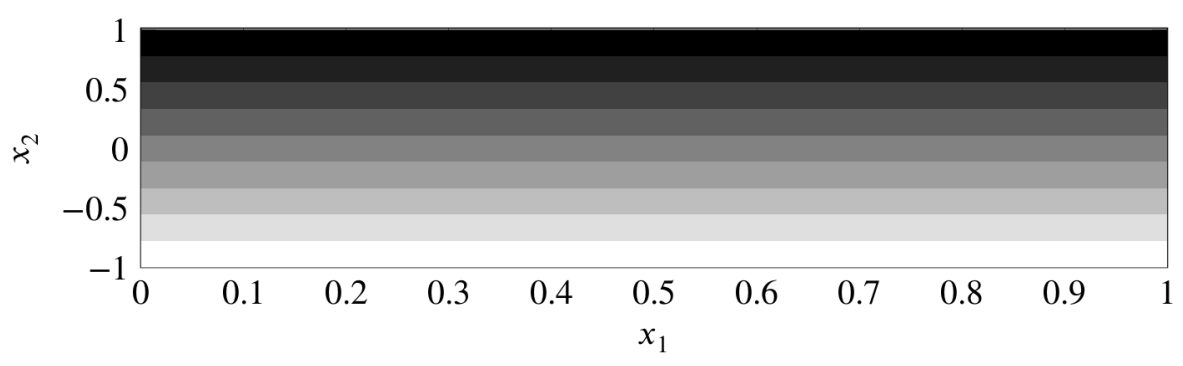

(b)

Figure 11: Reference image (a) and virtual image (b) in the virtual image coordinate system $\left(0, x_{1}, x_{2}\right)$.

- VIC settings: NURBS of order 3,6 points for the holes and B-Spline of order 3, 14 points for the notch, virtual image width $R=2$ pixels, the gray levels of the virtual image range from $f_{o}=128$, the average of the speckle (close to the boundaries) to $f_{b}=255$ for the background.

- Three VDIC settings: $\left(\alpha=0, c_{b}=10\right),\left(\alpha=0, c_{b}=-5\right),\left(\alpha=50, c_{b}=-5\right)$.

The VIC is used to measure the boundaries in both $F$ and $G$ images. As shown in Figure 10b it identifies well the border, despite the presence of defects. The two images, reference $F$ and virtual $G$, unwrapped in the virtual image coordinate system $\left(0, x_{1}, x_{2}\right)$, are presented in the Figure 11 As it can be shown, all along the boundary (expect for a few singularities due to defects), the gray level variation of the unwrapped reference image is close to the one of the virtual image. This shows that the retained B-Spline is able to describe the boundary shape.

The identification results in term of displacement norm without rigid body motion $\left\|U^{*}\right\|$ and in term of radial strain $\left|\varepsilon_{r r}\right|$ are shown in Figure 12 . These two terms are supposed to be null in case of rigid body displacement. For the radial strain, the results are calculated in a band of $2 L_{e}=100$ pixels. The results obtained with the unconstrained and unmasked VDIC $\left(\alpha=0, c_{b}=10\right)$, Figures $12 \mathrm{a}$ and $12 \mathrm{~b}$, show singularities in both displacement and strain fields, very close to the borders, which seem to be unphysical. Firstly, because the radial strain should be null due to rigid body movement and, secondly, because their size corresponds to the size of a finite element. The results obtained by the unconstrained and masked VDIC 


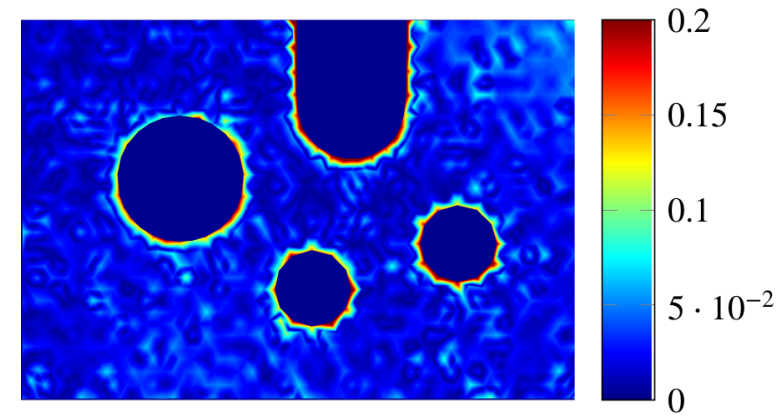

(a) $\left(\alpha=0, c_{b}=10\right)$

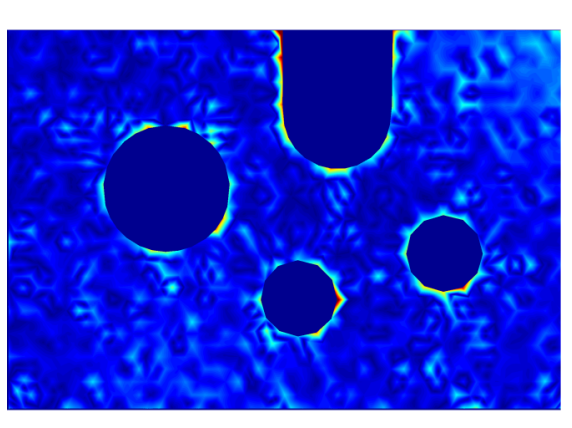

(c) $\left(\alpha=0, c_{b}=-5\right)$

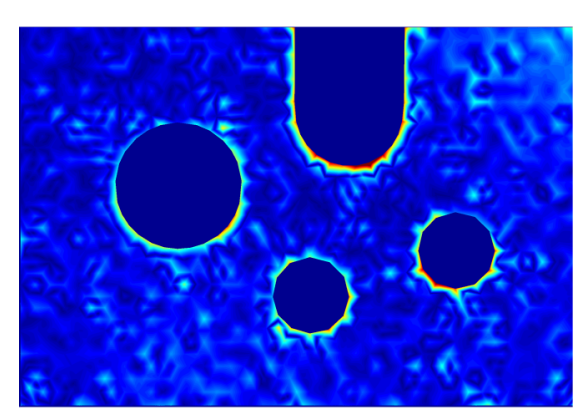

(e) $\left(\alpha=50, c_{b}=-5\right)$

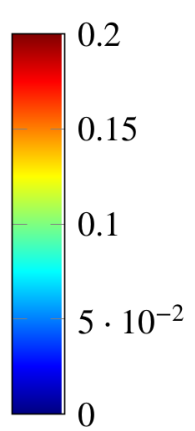

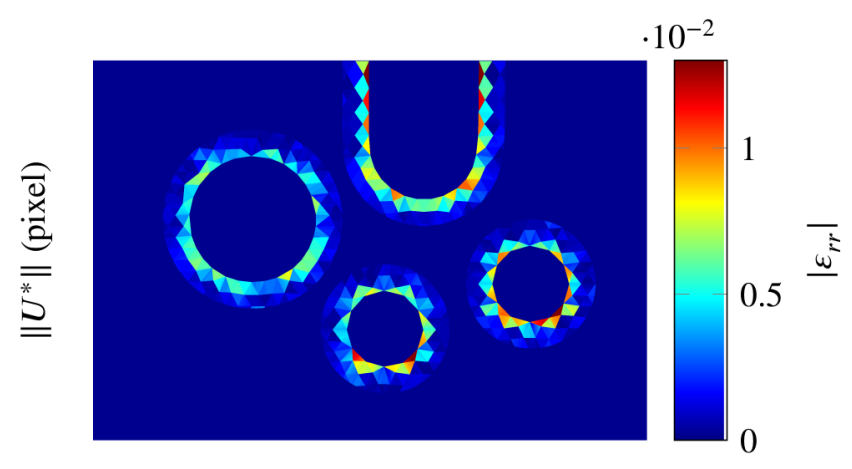

(b) $\alpha=0, c_{b}=10$ )

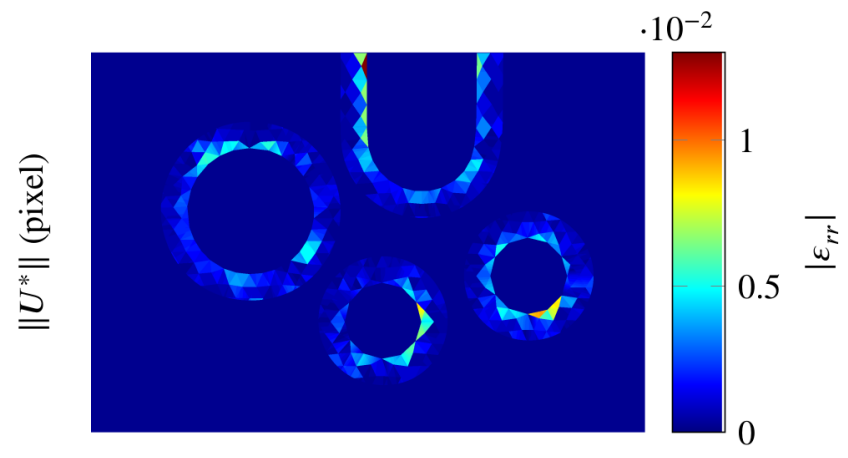

(d) $\left(\alpha=0, c_{b}=-5\right)$
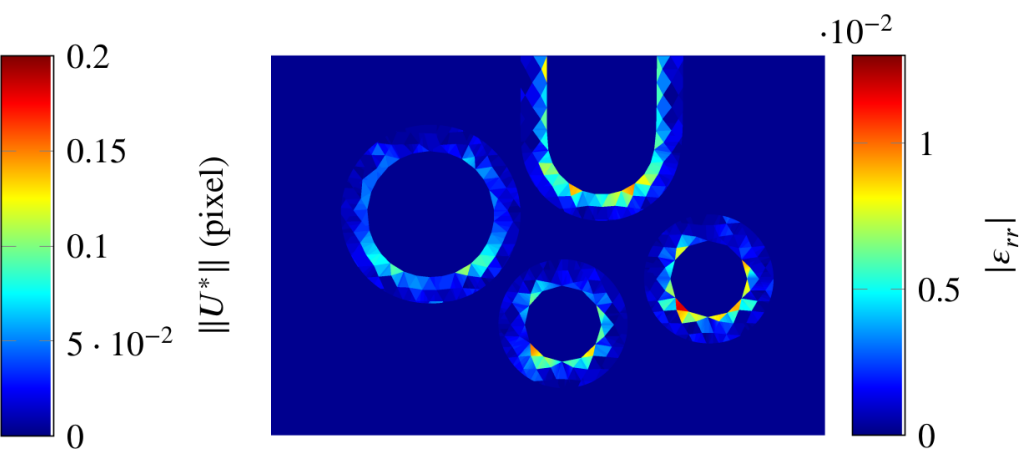

(f) $\left(\alpha=50, c_{b}=-5\right)$

Figure 12: Measured norm of the displacement without the rigid body displacement (left) and measured radial strains (right), for: unconstrained and unmasked VDIC ( $\left.\alpha=0, c_{b}=10\right)$ (top), unconstrained and masked VDIC $\left(\alpha=0, c_{b}=-5\right)$ (middle), and constrained and masked VDIC ( $\left.\alpha=50, c_{b}=-5\right)$ (bottom). 


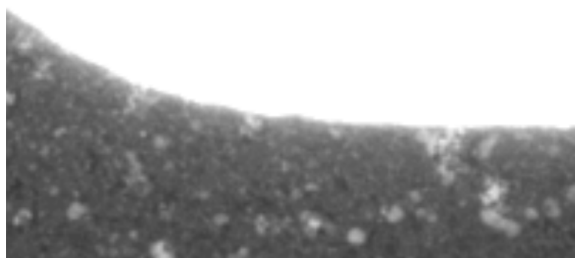

(a)

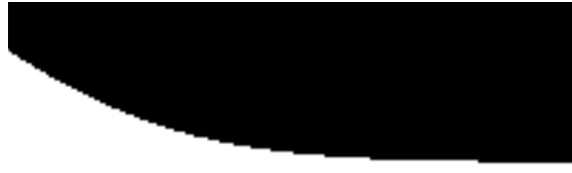

(b)

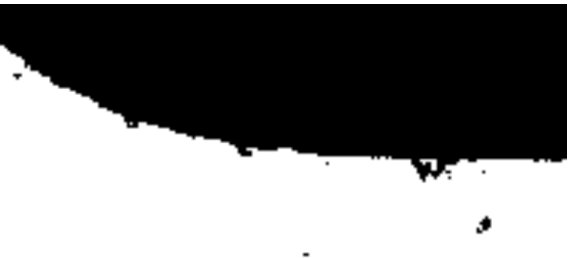

(c)

Figure 13: Zoom on a defect on the U shaped notch (a). Pixel mask used in VDIC (b) and mask created with simple threshold method (c).

$\left(\alpha=0, c_{b}=-5\right)$, Figures $12 \mathrm{c}$ and $12 \mathrm{~d}$, appear to be better because the above singularities are almost absent. For the constrained and masked DIC $\left(\alpha=50, c_{b}=-5\right)$, Figures $12 \mathrm{e}$ and $12 \mathrm{f}$ show again singularities along the borders. This is due to defects mentioned above: diffraction which affects the DIC close to the border and paint drops which distort the VIC measurement.

Figure 13 shows two types of masks related to the reference image of this experimental case. The first mask, Figure $13 \mathrm{~b}$, is created with the information on the position of the boundary and the second one, Figure 13c, with a simple threshold method based on gray levels. Due to the defects on the boundaries, the mask based on gray levels is less precise and regular than the one used in the VDIC method.

\section{Conclusion}

In this article, the boundary position measurement method (VIC) is used to improve the DIC precision on the sample boundary. The proposed VDIC method can be used at various levels. Firstly, in its unconstrained form, setting $\alpha=0$, it allows the creation of a perfectly adapted meshing whose nodes are precisely on the contour. Furthermore, depending upon the distance of exclusion $c_{b}$, the user may decide the creation of a pixel mask which is at a precise distance from the contour. Compared with classical threshold methods, this mask used the boundary position information to exclude unwanted pixels. As a consequence, the created mask is much more regular and precise. In the absence of optical or physical defects, the exclusion of a band of 1 pixel (corresponding to $c_{b}=-1$ ) is prescribed. For a synthetic performance test and an experimental study, this unconstrained and masked VDIC reduces significantly the displacement and strain measurement error on the boundary.

Moreover, in its constrained version, with $\alpha>0$, the VDIC imposes the finite element nodes to also belong to the boundary in the deformed state. This constraint is quite absolute as soon as $\alpha=50$. In case of absence of border defects, this constraint increases the precision of the radial displacement of a factor 2 .

For now the method is developed for small deformations thus no major change in the boundary shape. In case of creation of new boundary such as cracking or loss of matter, the curve equation as well as the finite element mesh should be defined from the final state. This will be investigated in a future work.

As shown in Figure 13, a classical preparation of the specimen for the DIC may lead to borders defects. The VIC part of the proposed method requires a careful border cleaning and a clean white background. The speckle has to range from half grey to black, which is sufficient for the DIC. Then, reducing (numerically) the image dynamics from white to half grey allow the VIC to be performed in optimal conditions. Ideally, the side of the specimen should be matte black painted to minimize the diffraction which slightly brightens the pixels close to the border (Figure 10b), in order to avoid DIC errors. This can be achieved by, firstly, 
painting in black the whole specimen and, secondly, applying the white speckle after having protected the sides.

\section{References}

[1] Besnard, G., F. Hild, and S. Roux, 2006: Finite-element displacement fields analysis from digital images: application to portevin-le chatelier bands. Experimental Mechanics, 46, no. 6, 789-803.

[2] Carroll, J. D., W. Abuzaid, J. Lambros, and H. Sehitoglu, 2013: High resolution digital image correlation measurements of strain accumulation in fatigue crack growth. International Journal of Fatigue, 57, 140150.

[3] Cheng, P., M. A. Sutton, H. W. Schreier, and S. R. McNeill, 2002: Full-field speckle pattern image correlation with B-spline deformation function. Experimental mechanics, 42, no. 3, 344-352.

[4] Fagerholt, E., T. Børvik, and O. Hopperstad, 2013: Measuring discontinuous displacement fields in cracked specimens using digital image correlation with mesh adaptation and crack-path optimization. Optics and Lasers in Engineering, 51, no. 3, 299-310.

[5] François, M.: Measurement uncertainty of the virtual image correlation method, submitted in Experimental Mechanics.

[6] François, M. L., 2016: Monitoring of debonding or cracking in bending tests by virtual image correlation. 8th RILEM International Conference on Mechanisms of Cracking and Debonding in Pavements, Springer, 739-745.

[7] Jiang, Z., J.-F. Witz, P. Lecomte-Grosbras, J. Dequidt, S. Cotin, C. Rubod, C. Duriez, and M. Brieu, 2017: Multiorgan motion tracking in dynamic magnetic resonance imaging for evaluation of pelvic system mobility and shear strain. Strain, 53, no. 2, e12224.

[8] Jiang, Z., J.-F. Witz, P. Lecomte-Grosbras, J. Dequidt, C. Duriez, M. Cosson, S. Cotin, and M. Brieu, 2015: B-spline based multi-organ detection in magnetic resonance imaging. Strain, 51, no. 3, 235-247.

[9] Luo, P., Y. Chao, M. Sutton, and W.-H. Peters, 1993: Accurate measurement of three-dimensional deformations in deformable and rigid bodies using computer vision. Experimental mechanics, 33, no. 2, $123-132$.

[10] Muskhelishvili, N. I., 1954: Some basic problems of the mathematical theory of elasticity. Springer Science \& Business Media.

[11] Passieux, J.-C. and R. Bouclier. 2019, Sur le solveur en corrélation d'images éléments finis, .

[12] Persson, P.-O. and G. Strang, 2004: A simple mesh generator in matlab. SIAM review, 46, no. 2, 329-345.

[13] Pierré, J.-E., J.-C. Passieux, J.-N. Périé, F. Bugarin, and L. Robert, 2016: Unstructured finite elementbased digital image correlation with enhanced management of quadrature and lens distortions. Optics and Lasers in Engineering, 77, 44-53.

[14] Réthoré, J. and M. François, 2014: Curve and boundaries measurement using b-splines and virtual images. Optics and Lasers in Engineering, 52, 145-155.

[15] Réthoré, J., F. Hild, and S. Roux, 2008: Extended digital image correlation with crack shape optimization. International Journal for Numerical Methods in Engineering, 73, no. 2, 248-272.

[16] Réthoré, J., 2018: Ufreckles. URL https://doi.org/10.5281/zenodo.1433776

[17] Semin, B., H. Auradou, and M. L. François, 2011: Accurate measurement of curvilinear shapes by virtual image correlation. The European Physical Journal-Applied Physics, 56, no. 1. 
[18] Sun, Y., J. H. Pang, C. K. Wong, and F. Su, 2005: Finite element formulation for a digital image correlation method. Applied optics, 44, no. 34, 7357-7363.

[19] Zhang, J., Y. Cai, W. Ye, and T. Yu, 2011: On the use of the digital image correlation method for heterogeneous deformation measurement of porous solids. Optics and lasers in engineering, 49, no. 2, 200-209. 